\title{
Zulliger e Habilidade Social: Evidências de Validade no Contexto Empresarial
}

\author{
Zulliger and Social Skills: Validity Evidences in Business Context
}

\author{
Jucelaine Bier Di Domenico Grazziotin \& Silvana Alba Scortegagna* \\ Universidade de Passo Fundo, Passo Fundo, Brasil
}

\begin{abstract}
Resumo
O objetivo deste estudo foi verificar evidências de validade do Zulliger no Sistema Compreensivo ZSC, focalizando a variável relacionamento, em correlação ao Inventário de Habilidades Sociais IHS. Participaram 19 sujeitos, entre 18 e 43 anos, de uma loja de supermercado. Nos indicadores, destacam-se as correlações das variáveis GHR, H, GPHR, Sum $H$ do ZSC com os fatores GIHS, F3 e F1 do IHS, e variáveis $A G, P H R$, Sum $T$ com os fatores GIHS e F5. Os resultados contribuíram para asseverar a validade do Zulliger no âmbito empresarial, e comprovaram a presença de um bom relacionamento interpessoal entre os profissionais que trabalham no atendimento ao público, confirmando as expectativas do estudo.

Palavras-chave: Validade, Zulliger, habilidade social, contexto empresarial.
\end{abstract}

\begin{abstract}
The aim of this study was to verify the validity evidence of Zulliger Comprehensive System ZSC focusing the variable 'relationship' in correlation to the Social Skills Inventory. Nineteen supermarket workers, aged between 18 and 43 years old, participated in the study. The indicators include the correlations of variables GHR, H, GPHR; Sum H of ZSC with the factors GIHS, F3 and F1 of HIS; and variables $A G$, $P H R$, Sum $T$ with the factors GIHS and F5.The results contributed to certify the validity of Zulliger in the business field and prove the presence of good interpersonal relationships among professionals who work with the public, confirming the assumptions of the study.

Keywords: Validity, Zulliger, social skills, business context.
\end{abstract}

A avaliação psicológica é uma prática frequente no contexto organizacional, em diversos ramos de atividade, pois possibilita melhor compreensão do funcionamento psicológico do indivíduo e contribui para a realização de procedimentos e encaminhamentos procedentes. Diante disso, a escolha de instrumentos adequados é de extrema importância (Godoy \& Noronha, 2005) e tem suscitado indagações na sociedade científica, referentes à qualidade, ao manuseio e aos resultados da avaliação (Pellini, 2008). Os questionamentos dos resultados de avaliações psicológicas, se, por um lado, demonstram a sua importância, estando inserida em vários contextos, por outro, apontam para alguns critérios técnicos e conceituais necessários para se qualificar um instrumento como adequado para uso.

Nessa perspectiva, um ponto fundamental relativo à cientificidade desses instrumentos é a sua validade. Historicamente, a validade tem sido atribuída ao teste que mensura realmente o que pretende medir (Anastasi \&

"Endereço para correspondência: Instituto de Filosofia e Ciências Humanas, Universidade de Passo Fundo, Curso de Psicologia, Campus Universitário, s/n, Prédio B-6, São José, Passo Fundo, RS, Brasil 99001-970. E-mail: jucelainegraz@terra.com.br e silvanalba@upf.br
Urbina, 2000), sendo verificada, basicamente, por estudos de validade de conteúdo, critério e construto. Entretanto, essa definição foi aprimorada, quando da revisão dos Standards para a versão 1999 (American Educational Research Association [AERA], American Psychological Association [APA], \& National Council for Measurement in Education [NCME], 1999). Nesse novo documento, validade "refere-se ao grau em que evidência e teoria sustentam as interpretações dos escores dos testes vinculados aos usos propostos dos testes" (p. 9).

O processo de validação, portanto, reúne um conjunto de evidências que asseguram a relevância do teste, a sua utilidade nos usos propostos e as interpretações geradas. Nesse sentido, superados os critérios mínimos para o uso dos testes psicológicos instituídos na resolução $n^{\circ}$ 002/ 2003 do Conselho Federal de Psicologia (CFP, 2003), atualmente, não se valida o teste em si, mas as interpretações propostas por este e as aplicações práticas pretendidas (Primi, Muniz, \& Nunes, 2009).

Considerando que, nas empresas, a seleção de pessoal é uma prática frequente, as escolhas dos instrumentos, sejam eles entrevistas, testes de habilidades cognitivas, ou, ainda, de personalidade, além de responder aos objetivos almejados, devem ser realizadas por especialistas com domínio, tanto no uso dos instrumentos como das 
teorias que os fundamentam. Sendo assim, cada instrumento deve contribuir e auxiliar as demais medidas psicológicas usadas, e a combinação desses elementos são imprescindíveis na inferência e tomada de decisão para, por exemplo, predizer o desempenho. Por essa razão, Chiavenato (2002) considera que a utilização de Métodos Projetivos de avaliação da personalidade, assim como de testes psicométricos, podem incrementar a qualidade dos resultados obtidos nesse contexto, desde que sejam válidos e precisos.

Nesse sentido, a literatura reporta que os Métodos Projetivos constituem um desafio à parte nesse campo de investigação, uma vez que a análise e a interpretação de seus resultados encontram-se amparadas mais em pressupostos teóricos do que em evidências psicométricas. Se essa é uma de suas características, também é verdade que sua natureza permite $o$ acesso de forma mais indireta à investigação da personalidade, o que possibilita o alcance de respostas menos manipuláveis e evita a interferência do fator desejabilidade social, tão frequente em situações de avaliação compulsória no setor empresarial (Villemor-Amaral, 2009).

É interessante notar que, no contexto organizacional, a desejabilidade social encontra-se fortemente presente. Como os dados obtidos nos testes empregados têm impacto direto sobre os resultados na seleção de pessoal, no desenvolvimento da carreira, é comum que os examinandos procurem parecer melhores do que são, respondendo da maneira que julgam mais condizente com as expectativas do empregador (Dilchert, Ones, Visweswaran, \& Deller, 2006; Meade, 2004).

Tal constatação reforça o interesse para o uso de instrumentos projetivos no âmbito empresarial. Entre as técnicas confiáveis e recomendáveis, destaca-se o Zulliger que, dependendo do sistema utilizado, permite tanto a aplicação individual como coletiva (Villemor-Amaral, 2005, 2009; Villemor-Amaral \& Primi, 2009).

O Zulliger, especialmente no Sistema Compreensivo ZSC, forma individual, integra diversos aspectos da personalidade, com base tanto nos preceitos da psicometria, como nos da projeção, o que permite uma investigação mais completa do universo psíquico do examinando, tão solicitada no campo empresarial (Villemor-Amaral \& Primi, 2009). Dessa forma, a importância em se adaptar, normatizar e validar esse sistema tem sido salientada por Zdunic (2003), ao perceber, em sua prática profissional que, após meses e anos da entrega dos relatórios, diversas empresas referiram que os prognósticos das avaliações de pessoal haviam se cumprido, o que, além de conferir confiabilidade ao instrumento como preditor do desempenho no trabalho, incrementa a necessidade de estudos nessa área.

Assim como a referida autora, na Argentina, pesquisadores de outros países empenham-se em demonstrar o uso do ZSC, inclusive com indivíduos de todas as faixas etárias. Destacam-se, Mahmood (1990), na Grã-Bretanha; Zennaro e Lis (1998) na Itália; onde aparecem os primei- ros trabalhos. No Brasil, os estudos ainda são raros e recentes, e apenas com a população de adultos. Entre os nacionais, Villemor-Amaral e Lamounier (2006) investigaram a convergência, quanto ao tipo de vivência (EB), entre o ZSC e o Rorschach no Sistema Compreensivo RSC, que consiste num fator de grande relevância entre essas técnicas. As autoras descobriram coincidências significativas com relação aos tipos introversivo, extratensivo e evitativo, mas não encontraram concordância entre as técnicas para os ambiguais. Essa discordância foi atribuída ao número diferenciado de pranchas entre as técnicas, o qual leva a uma menor quantidade de respostas e de determinantes. Embora se constate a necessidade de otimização do tipo de vivência (EB) no ZSC, os dados foram considerados favoráveis com as hipóteses testadas.

A fim de investigar a validade do ZSC no contexto clínico, foram realizados dois estudos, um para avaliar a depressão (Villemor-Amaral \& Machado, 2008), com a utilização dos indicadores da constelação DEPI (Índice de Depressão); e o outro na investigação do Transtorno Obsessivo Compulsivo (TOC; Pianowski \& VillemorAmaral, 2008). Em ambos os estudos, as autoras utilizaram grupos contrastantes de comparação, um de pacientes e outro de não pacientes. Os resultados foram considerados satisfatórios, na medida em que sugeriram que os indicadores de depressão e sintomas de transtorno obsessivo compulsivo no RSC podem se manifestar também no ZSC.

Outra pesquisa de validade do ZSC, mas específica para o contexto empresarial, foi desenvolvida por Ferreira e Villemor-Amaral (2005). As autoras buscaram a correlação do ZSC com um questionário de avaliação de desempenho, específico da empresa, de profissionais com formação em Ciências Exatas. A avaliação considerou os itens: Relacionamento interpessoal, tomada de decisões, busca por inovação, organização e solução de problemas. As análises de correlação indicaram que profissionais mais disciplinados e organizados, com iniciativa, visão prática, e que visam a resultados concretos são melhor avaliados do que os imaturos emocionalmente, altamente críticos e mais egocêntricos, que priorizam as próprias necessidades em detrimento das de outros. Assim, o ZSC demonstrou potencial para apreender os aspectos de personalidade associados ao desempenho no trabalho.

Nessa direção, Vaz (2000) desenvolveu um estudo das questões relativas à atividade profissional, interação social e personalidade, utilizando o Zulliger no sistema Klopfer em 15 categorias profissionais, sendo discutida a questão do background cultural. $\mathrm{O}$ autor destaca não ser tarefa simples avaliar a personalidade tanto para seleção, como para psicotécnico ou perícia judicial, pois parâmetros quantitativos construídos por meio de uma técnica ou teste são elementos objetivos que representam realidades do interior de cada sujeito (Vaz, 2002).

Portanto, muitas são as variáveis e as questões que cercam a avaliação psicológica em atividades empresariais. 
Como pode ser observado, a inserção do ZSC neste processo deve atravessar as considerações sobre demandas da empresa, do avaliado, do psicólogo responsável e de outros instrumentos válidos para essa finalidade (Zdunic, 2003). Dessa forma, as dificuldades para validação das técnicas projetivas são demonstradas em diversos trabalhos, devido à complexidade de avaliar os fenômenos psíquicos envolvidos na dinâmica de personalidade.

Recentemente, Franco e Villemor-Amaral (2009), preocupadas com a dificuldade de validação de técnicas projetivas, realizaram três estudos de convergência entre o ZSC e o Pfister. O primeiro, de validade de critério, buscou verificar a validade das Constelações do ZSC. O segundo, de validade convergente, correlacionou os indicadores afetivos e cognitivos dos Testes ZSC e Pfister. E o terceiro verificou a validade do raciocínio clínico, comparando a convergência ou divergência de protocolos de mesmos indivíduos. Os participantes foram subdivididos em dois grupos, um composto por não pacientes e o outro composto por pacientes psiquiátricos. As autoras concluíram que o ZSC oferece uma compreensão detalhada sobre alguns aspectos da personalidade e que o uso de estratégias diferentes de validação de instrumentos pode ampliar as possibilidades de compreensão das técnicas projetivas no que se refere aos seus alcances e limites.

Finalmente, os estudos de normatização para o ZSC no Brasil, foram desenvolvidos por Franco, Cardoso, Villemor-Amaral e Primi (2009), mas não foi possível estabelecer a nota Z. Desta forma, dando prosseguimento às pesquisas de validade do $\mathrm{ZSC}$, e com o objetivo de complementar o que foi realizado até então, Santana, Cardoso e Villemor-Amaral (2011) procuraram instituir a nota $Z$, em termos de frequência (Zf) e soma (Zsum). As autoras ressaltaram a relevância desse estudo para informar o funcionamento da atividade organizativa do sujeito.

Observa-se que no Brasil, nos últimos anos, os estudos com o ZSC tiveram avanços significativos, mas a necessidade de progresso é ainda muito grande. Notadamente, no contexto empresarial, em que os processos de seleção de pessoal são realizados quase exclusivamente com testes de personalidade, evidencia-se uma carência de pesquisas dos fatores preditores de traços de personalidade em relação ao desempenho do sujeito no trabalho (Baumgartl \& Primi, 2006).

De outra maneira, esta preocupação também é demonstrada por A. Del Prette e Del Prette (2006). Os autores referem que indivíduos com bom relacionamento interpessoal são mais produtivos no trabalho, destacando, ainda, a utilização de outro instrumento confiável para a avaliação nesse contexto, o Inventário de Habilidades Sociais (IHS; A. Del Prette \& Del Prette, 2005).

Bandeira, Costa, Z. A. P. Del Prette, Del Prette e GerkCarneiro (2000) realizaram uma pesquisa investigativa das qualidades psicométricas do IHS, em termos de sua precisão, com estudantes de psicologia. A fidedignidade do IHS foi avaliada por meio de uma análise correlacional entre os escores de duas aplicações, teste-reteste, confirmando uma correlação alta e significativa $(r=0,90, p=0$, 001) entre as duas aplicações. A validade concomitante do IHS foi avaliada através de uma análise correlacional entre os dados do IHS e a escala de assertividade de Rathus que mensuram construtos correlatos. Os resultados demonstraram correlação significativa entre as duas escalas $(r=0,79, p=0,001)$, e uma correlação mais elevada $(r=0,81, p=0,001)$ foi obtida entre o IHS e os vinte itens da escala de Rathus que compõem o seu principal fator.

Especificamente, no contexto organizacional, realizou-se a análise do repertório de habilidades sociais em profissionais de uma empresa em fusão, utilizando-se do IHS (Silva, 2004). O estudo, além de demonstrar a importância das habilidades sociais e a necessidade de maiores pesquisas nesta área, destacou a existência de várias definições desse construto, mesmo que em todas prevaleça a compreensão de que ter habilidades sociais implica apresentar respostas que tenham alta probabilidade de fortalecer, promover ou intensificar as relações interpessoais. Acrescenta, ainda, que investir no desenvolvimento de habilidades sociais dos funcionários pode aumentar tanto seu bem estar, como a produtividade da organização em um contexto flexível, de contínua e rápida modificação.

Desse modo, sendo essas habilidades preditoras de um bom desempenho em diversas funções no trabalho, buscam-se verificar as "habilidades sociais" no ZSC por meio de evidência de validade, correlacionando as variáveis relacionamento do ZSC com os fatores do IHS Cabe destacar que o ZSC é um dos instrumentos que reúne tanto conceitos de projeção como de psicometria que avalia o relacionamento interpessoal, ou seja, as representações que o indivíduo tem sobre si e sobre o outro (Villemor-Amaral \& Primi, 2009). Por outro lado, o Inventário de Habilidades Sociais (IHS; A. Del Prette $\&$ Del Prette, 2005) configura-se em um teste notoriamente psicométrico que avalia um conjunto de desempenhos em que a pessoa apresenta maior ou menor competência social. Assim, embora haja diferenças na construção dos instrumentos, ambos avaliam construtos relacionados.

Portanto, constitui-se objetivo deste estudo a busca por evidências de validade para um conjunto de variáveis do relacionamento no ZSC, a saber: SumT (sombreado textura); $F d$ (comida ou ação de comer); $H$ (humano inteiro), $H d$ (detalhe humano), $(H)$ (para-humano inteiro), $(H d)$ (detalhe para-humano); GHR (boa representação humana) e $P H R$ (representação humana pobre); $a$ (movimento ativo) e $p$ (movimento passivo); (isolamento); PER (resposta personalizada); $A G$ (movimento agressivo); $C O P$ 
(movimento cooperativo) que, por hipótese, estariam correlacionadas com os fatores do IHS: (F1) autoafirmação e enfrentamento com risco; (F2) autoafirmação na expressão de afeto positivo; (F3) conversação e desenvoltura social; (F4) autoexposição a desconhecidos e situações novas; (F5) autocontrole da agressividade em situações aversivas. Especificamente, pretende-se verificar se as variáveis COP, GHR, GPHR, a, Sum $H$, $H$ que podem informar sobre o bom relacionamento e a percepção interpessoal adequada se correlacionam positiva e significativamente com fatores do IHS; e se as variáveis $A G, P H R, p, F d$, Sum $T$, PER, isolamento, que podem informar sobre o relacionamento e percepção interpessoal prejudicada se correlacionam negativa e significativamente com os cinco fatores do IHS.

\section{Método}

\section{Participantes}

Foram participantes 19 sujeitos, entre 18 e 43 anos de idade, média 27,5 anos $(D P=8,83)$, dez $(53 \%)$ do gênero feminino e nove (47\%) do gênero masculino, 15 (78,94\%) com ensino médio completo e quatro $(21,05 \%)$ ensino médio incompleto, de uma loja de supermercados. Os participantes foram selecionados de forma intencional tendo como critério de inclusão os que exerciam atividades de atendimento direto ao cliente. Destes, sete $(36,84 \%)$ na função de operadores e frente de caixa; sete $(36,84 \%)$ como atendentes de perecíveis e cinco $(26,31 \%)$ no cargo de atendentes de loja. O tempo de permanência na mesma atividade e local de trabalho variou entre um a oito anos.

\section{Instrumentos}

Zulliger, no Sistema Compreensivo ZSC - forma individual (Villemor-Amaral \& Primi, 2009). Composto de três lâminas com manchas de tinta, constitui-se em um teste que possibilita obter informações sobre o funcionamento psíquico do individuo, amparado tanto nos conceitos de psicometria, quanto nos de projeção. Quanto à confiabilidade do teste, foram realizados dois estudos de precisão, um empregando o reteste e o outro analisando a precisão do avaliador. No delineamento teste-reteste, a maioria dos indicadores obteve valores acima de 0,70 ( $p=0,001)$; e os resultados de concordância entre os juízes avaliadores obtiveram correlações entre $0,60-0,80$, indicando uma associação alta (Muniz, Machado, VillemorAmaral, \& Primi, 2009). A aplicação do teste consiste em duas etapas, a fase da associação e a do inquérito. $\mathrm{Na}$ associação, o sujeito deve responder a questão 'com que isso se parece'. Já, na fase do inquérito é importante verificar-se 'onde foi que a pessoa viu' e 'o que na mancha fez com que parecesse aquilo' que foi dito.

Inventário de Habilidades Sociais (IHS; A. Del Prette $\&$ Del Prette, 2005). É um instrumento de autorrelato com 38 itens o qual possibilita a avaliação das habilidades sociais em diferentes contextos. Trata-se de um teste psicométrico com bom padrão de confiabilidade e de consistência interna (alfa de Cronbach $=0,75$ ); estabilidade teste-reteste $(r=0,90 ; p=0,001)$ e validade concomitante com o Inventário de Rathus $(r=0,79 ; p=0,01)$. Embora tenha sido validado, inicialmente na população universitária, o IHS Del Prette tem sido utilizado na avaliação de desempenho de adultos com formação de segundo e terceiro graus.

Nas instruções do IHS, solicita-se ao respondente que estime a frequência com que reage a uma situação descrita em cada item, em uma escala tipo Likert, que varia de 0 (nunca ou raramente) a 4 (sempre ou quase sempre) e que avalia cinco fatores relacionados à Habilidade Social. Este inventário mostra-se útil na seleção de pessoal, na redistribuição de função, e na gestão de recursos humanos, levando-se em consideração tarefas e competências.

\section{Procedimentos}

Após a aprovação do estudo pelos dirigentes da empresa e pelo Comitê de Ética em Pesquisa (n. 076/2009), iniciaram-se os procedimentos para a coleta de dados. O contato com o responsável pelo departamento de pessoal de uma das lojas, recomendada pelos dirigentes da empresa, possibilitou a indicação dos participantes desse estudo. Assim sendo, todos os funcionários, responsáveis por setores de atendimento direto ao publico do supermercado, ou seja, operadores e frente de caixa; atendentes de perecíveis e de loja; foram convidados a participar do estudo, e aceitaram ao convite.

Aos participantes da pesquisa, inicialmente, foram expostos os objetivos e os procedimentos do trabalho, e, na sequência, mediante a assinatura do Termo de Consentimento Livre e Esclarecido, procedeu-se à explicação das instruções de cada instrumento de avaliação, garantindolhes o anonimato e, ainda, o esclarecimento de eventuais questões. A coleta de dados foi realizada pela autora do trabalho nas dependências da empresa. Os participantes responderam ao ZSC seguido pelo IHS, de forma individual em uma única sessão de aproximadamente duas horas para cada individuo.

$\mathrm{Na}$ descrição dos resultados, primeiramente são apresentadas as estatísticas descritivas para caracterizar as variáveis. Posteriormente, diante do objetivo principal do estudo, qual seja, buscar evidência de validade convergente para o ZSC com base na comparação com o IHS, utilizou-se a prova de correlação de Pearson. Os cálculos foram realizados utilizando-se o programa SPSS, versão 11.5. Para as variáveis do ZSC foram observadas as expectativas normativas do grupo de não pacientes (Villemor-Amaral \& Primi, 2009) e para o IHS foram considerados os índices normativos de A. Del Prette e Del Prette (2005). 
Grazziotin, J. B. D. D. \& Scortegagna, S. A. (2012). Zulliger e Habilidade Social: Evidências de Validade no Contexto Empresarial.

\section{Resultados}

Em um primeiro momento, serão apresentadas as estatísticas descritivas das variáveis de relacionamento do ZSC e, na sequência, as do IHS. Por fim, apresentam-se as análises de correlação para verificar as evidências de validade obtidas pelo teste estatístico de Pearson. Dessa forma, na Tabela 1, podem ser visualizadas as estatísticas descritivas do ZSC.

Tabela 1

Estatísticas Descritivas da Variável Relacionamento do ZSC

\begin{tabular}{lccccccccc}
\hline Zulliger & $N$ & Mínimo & Máximo & Média & Mediana & $D P$ & 25 & 50 & 75 \\
\hline COP & 19 & 0 & 2 & 0,53 & 0,00 & 0,61 & 0 & 0 & 1,00 \\
$A G$ & 19 & 0 & 2 & 0,37 & 0,00 & 0,68 & 0 & 0 & 1,00 \\
GHR & 19 & 0 & 3 & 1,26 & 1,00 & 0,87 & 0 & 1 & 2,00 \\
PHR & 19 & 0 & 3 & 1,00 & 1,00 & 0,88 & 0 & 1 & 1,00 \\
GPHR & 19 & -2 & 3 & 0,26 & 0,00 & 1,15 & 0 & 0 & 2,00 \\
$a-p$ & 19 & -1 & 5 & 1,11 & 1,00 & 1,76 & -1 & 1 & 2,00 \\
Fd & 19 & 0 & 2 & 0,16 & 0,00 & 0,50 & 0 & 0 & 0 \\
SumT & 19 & 0 & 2 & 0,53 & 0,00 & 0,77 & 0 & 0 & 1,00 \\
SumH & 19 & 0 & 6 & 1,89 & 1,00 & 1,56 & 1 & 1 & 2,00 \\
$H$ & 19 & 0 & 3 & 0,84 & 1,00 & 0,90 & 0 & 1 & 1,00 \\
PER & 19 & 0 & 2 & 0,74 & 1,00 & 0,81 & 0 & 1 & 1,00 \\
Isolamento & 19 & 0 & 0,60 & 0,28 & 0,30 & 0,15 & 0,20 & 0,30 & 0,40 \\
\hline
\end{tabular}

Na Tabela 1, pode-se observar que os participantes obtiveram uma média elevada em $\operatorname{SumT} 0,53(D P=0,77)$ e uma média baixa no índice de isolamento $0,28(D P=0,15)$, considerando os parâmetros normativos para SumT 0
$(D P=0,2)$ e para o índice de isolamento $1,3(D P=1,6)$. As demais variáveis de relacionamento do ZSC apresentaram-se entre as expectativas normativas. A Tabela 2 apresenta as estatísticas descritivas do IHS.

Tabela 2

Estatísticas Descritivas dos Fatores do IHS

\begin{tabular}{lccccccccc}
\hline & & & & & & \multicolumn{3}{c}{ Percentil } \\
IHS & $N$ & Mínimo & Máximo & Média & Mediana & $D P$ & 25 & 50 & 75 \\
\hline GIHS & 19 & 8 & 90 & 50,3 & 53 & 26,5 & 28 & 53 & 78 \\
F1IHS & 19 & 8 & 98 & 45,37 & 43 & 22,45 & 23 & 43 & 63 \\
F2IHS & 19 & 1 & 98 & 52,29 & 52,5 & 31,65 & 22,5 & 52,5 & 87,5 \\
F3IHS & 19 & 2 & 92,5 & 59,39 & 68 & 31,36 & 33 & 68 & 88 \\
F4IHS & 19 & 4 & 96 & 45,65 & 42,5 & 26,75 & 27,5 & 42,5 & 67,5 \\
F5IHS & 19 & 22,5 & 87,5 & 64,6 & 67,5 & 19,95 & 47,5 & 67,5 & 82,5 \\
\hline
\end{tabular}

Verifica-se, por meio da Tabela 2, que os participantes obtiveram um escore geral de Habilidades Sociais - GIHS na média, indicando bom repertório nessa habilidade, sendo que houve uma variação do percentil mínimo de 8 , baixo repertório, e máximo de 90 , repertório elaborado. Quanto aos fatores individuais, pôde ser observado que o F1, Enfrentamento Com Risco, apresentou percentil 43 Médio Inferior MI, considerado bom repertório. O F2, Auto Afirmação na Expressão de Afeto Positivo, percentil 52 , na média $M$, configurando-se num bom repertório. $\mathrm{O}$ F3, Conversação e Desenvoltura Social, percentil 68 Médio Superior MS, repertório elaborado. O F4, Auto
Exposição a Desconhecidos ou a Situações novas, percentil 42, repertório Médio Inferior, um bom repertório e o F5, Auto Controle da Agressividade em Situações Aversivas, percentil 67,5, Médio Superior MS, repertório elaborado. A Tabela 3 apresenta as correlações das variáveis de relacionamento do ZSC e do IHS.

Verifica-se na Tabela 3 que todas as variáveis relacionamento do ZSC apresentaram correlação positiva ou negativa com os fatores do IHS, tanto com o fator geral (GIHS), como com os individuais (F1, F2, F3, F4, F5). Entre as correlações, observa-se que GPHR e SumH apresentaram correlações positivas com GIHS, F3 e F1 res- 
Tabela 3

Correlações de Pearson das Variáveis Relacionamento do ZSC com Fatores do IHS

\begin{tabular}{|c|c|c|c|c|c|c|}
\hline & GIHS & F1IHS & F2IHS & F3IHS & F4IHS & F5IHS \\
\hline$C O P$ & 0,05 & & & 0,21 & & \\
\hline$A G$ & $-0,36$ & & & & & $-0,49^{*}$ \\
\hline GHR & 0,34 & 0,36 & & 0,35 & & \\
\hline PHR & $-0,33$ & 0,25 & & $-0,35$ & & $-0,35$ \\
\hline GPHR & $0,51 *$ & & & $0,54 *$ & & 0,33 \\
\hline$a-p$ & $-0,24$ & & & $-0,21$ & & $-0,23$ \\
\hline$F d$ & 0,39 & 0,24 & & & 0,23 & 0,22 \\
\hline Sum $T$ & $-0,15$ & 0,26 & 0,30 & & $-0,24$ & $-0,53 *$ \\
\hline Sum H & 0,28 & $0,53^{*}$ & & 0,32 & & 0,20 \\
\hline Pure $H$ & 0,15 & & & $0,42 *$ & & 0,36 \\
\hline PER & 0,03 & & & $-0,21$ & 0,26 & \\
\hline Isolamento & $-0,20$ & & & 0,36 & & \\
\hline
\end{tabular}

* Correlação significativa no nível de $p<0,05$.

pectivamente. Assim, em uma mesma direção, se GPHR e SumH aumentam, os fatores de GIHS, F3 e F1 tendem a aumentar. Contrariamente, $A G$ e Sum $T$ apresentaram correlações negativas. Desse modo, observa-se que, em direções opostas, se uma variável aumenta, a outra tende a diminuir. Então, de acordo com a tabela, as correlações das variáveis $A G$ e GIHS, assim como $A G$ e $\mathrm{F} 5$ foram negativas. Nesse caso, pode-se dizer que, se uma pessoa tiver alto escore em GIHS ou F5, tenderá a baixar em $A G$. Da mesma forma, com a variável $\operatorname{Sum} T$, se houver um alto escore em F5, o escore tenderá a ser baixo em SumT.

\section{Discussão}

Em relação ao teste ZSC, a maioria das variáveis relacionamento COP, $A G$, GHR, PHR, GPHR, a, p, Fd, SumH, $H$, mantiveram-se na média normativa (Villemor-Amaral \& Primi 2009), apenas as variáveis SumT e Isolamento oscilaram entre os parâmetros normativos. Estes primeiros achados são importantes à medida que acenam para a validade do ZSC para mensurar aspectos do relacionamento interpessoal, no âmbito empresarial.

Nesse sentido, as respostas de movimento cooperativo COP identificam atitudes e expectativas positivas em relação ao mundo real e uma percepção do meio menos hostil ou agressiva. Já, as respostas de movimento agressivo AG, para Zdunic (2003), demonstram uma atitude hostil frente aos outros, apesar de, em alguns postos de trabalho, poder ser útil a presença de componentes agressivos, os quais têm de estar acompanhados de outros fatores relacionados a um bom controle, para o resultado de uma conduta adaptativa. De qualquer forma, é útil comparar a quantidade de respostas $\mathrm{COP}$, com a quantidade de conteúdos AG. No presente estudo, além destas variáveis terem permanecido nos índices normativos, houve uma incidência maior de COP do que $\mathrm{AG}$, o que sugere algo positivo em relação ao grupo estudado e, tam- bém, esperado, ao se tratar de pessoas que trabalham com atendimento direto ao público.

As qualidades gerais das percepções e representações humanas e interpessoais são atribuídas no Zulliger pelas respostas GHR e PHR. As respostas GHR remetem a percepções ou representações de si mesmo, do outro, e as relações manifestadas em verbalizações humanas precisas, realistas, lógicas, intactas e interações boas ou cooperativas. As respostas $P H R$ são percepções negativas ou problemáticas como aquelas manifestadas em representações ou percepções distorcidas, irrealistas, danificadas, confusas, ilógicas, agressivas, ou más (Viglione, Perry, Jansak, Meyer, \& Exner, 2003).

GPHR é uma variável que sinaliza a incidência da prevalência das respostas boas (GHR) sobre as más (PHR; Franco, Cardoso, Villemor-Amaral, \& Primi, 2009). Espera-se que pessoas adultas, bem adaptadas, e com capacidade para ver a si mesmo e aos demais de modo integrado e realístico, apresentem $G H R>P H R$ (Villemor-Amaral \& Primi, 2009). Em decorrência de a amostra ser constituída por pessoas que trabalham em comércio e atendimento direto ao público, esperava-se encontrar uma boa proporção de respostas $G H R$ e um menor índice de respostas $P H R$ e que, comparativamente ao IHS, os que apresentassem maior índice nos escores oferecessem maior índice de respostas GHR. Este aspecto mostrou ter-se confirmado.

É importante enfatizar que os escores de conteúdo sugerem principalmente focos de atenção da pessoa, suas preocupações e, principalmente, sua amplitude e predominância de interesse. Assim, de forma geral, respostas de conteúdo humano $H,(H)+H d+(H d)$ indicam interesse pelos outros, e, dependendo de sua distribuição e características, revelarão facilidades ou dificuldades nos relacionamentos interpessoais. O predomínio de respostas de perceptos humanos inteiros $H$ sobre outros conteúdos humanos $(H)+H d+(H d)$ favorece o bom ajus- 
tamento psicológico e fornece ao indivíduo uma impressão clara e coerente sobre si mesmo (Weiner, 2000). Considerando os resultados do presente estudo, tanto o índice de respostas $H$ puro, como Sum $H[(H)+H d+(H d)$ apresentaram-se nos índices normativos.

A variável de sombreado SumT expressa a necessidade de contato e, quando aumentada, revela a tendência de a pessoa experimentar sentimentos de solidão além do esperado. $\mathrm{O}$ exagero dessa variável pode indicar contatos pessoais excessivamente pegajosos e aversivos (VillemorAmaral \& Primi, 2009). Os índices dessa variável, na amostra pesquisada, mantiveram-se um pouco acima 0,5 $(D P=0,77)$, ou seja, por ser pouco frequente, esperam-se valores $0(D P=0,2)$. Este aumento pode ser devido ao tipo de função realizada, de atendimento ao cliente, demonstrando que as pessoas que exercem tais atividades necessitam de maior contato e de um reconhecimento pelo desempenho mais explícito (Zdunic, 2003).

De forma semelhante, a variável isolamento que representa o isolamento social, o retraimento (Villemor-Amaral \& Primi, 2009), apresentou índices mais baixos do que os estabelecidos nos parâmetros normativos. Aqui, também, aventou-se a hipótese desse resultado estar relacionado às exigências da função exercida pela amostra estudada, ou seja, de pessoas que se direcionam para um maior contato social. Observa-se, assim, que tanto as variáveis do relacionamento no $\mathrm{ZSC}$, como os fatores do IHS, mantiveram-se nos índices normativos. Desse modo, torna-se importante discutir os escores dos fatores do IHS, já que vem ratificar os resultados do ZSC e as interpretações dos escores deste instrumento.

Para A. Del Prette e Del Prette (2005), entre os aspectos importantes que devem ser considerados na análise das habilidades sociais é a sua circunstancialidade. Este aspecto pode ser verificado no grupo estudado, pois, de acordo com a tabela geral do IHS (Tabela 2), percebe-se que, apesar de o grupo apresentar um repertório situado na média, foram evidenciadas características individuais nos fatores.

No F1, por exemplo, o grupo apresentou bom repertório, mas ficou um pouco abaixo da média, isso pode revelar que este grupo específico da área de atendimento ao cliente não apresenta um repertório tão elaborado no enfrentamento com risco, bem como no F4, autoexposição a desconhecidos e situações novas. Em contrapartida, no $\mathrm{F} 3$, que seria a conversação e desenvoltura social e no F5 que é o autocontrole da agressividade em situações aversivas, demonstrou repertório elaborado. Para ter um bom desempenho pessoal e profissional, nas relações interpessoais, as pessoas precisam desenvolver habilidades sociais que lhes ajudem a enfrentar os problemas ocorridos (Silva, 2004).

É esperado que um grupo que trabalha com clientes, na função de atendimento, apresente um bom repertório de habilidades sociais e demonstre em alguns fatores um repertório bem elaborado, principalmente aqueles que se relacionam com suas principais tarefas. Em ambientes organizacionais, refere A. Del Prette e Del Prette (2005), a identificação de áreas de maior ou menor competência social, assim como os itens específicos que se apresentam como mais elaborados ou como deficitários, permite uma caracterização do perfil sócio comportamental do respondente, o que pode ser particularmente útil na seleção de pessoal para determinadas tarefas e na redistribuição de funções.

Outro aspecto relevante a ser considerado em relação ao IHS é que a amostra pesquisada apresenta um nível de escolaridade situado entre ensino médio completo $(78,94 \%)$ e ensino médio incompleto $(21,05 \%)$. No entanto, os índices pesquisados na tabela normativa referem-se a uma amostra de nível universitário, sendo assim, poderiam obter-se índices maiores na presente amostra, se fosse correlacionada com os seus respectivos níveis de escolaridade.

No que se refere à correlação das variáveis de relacionamento do ZSC com os fatores do IHS (Tabela 3), observou-se que houve correlações significativas entre os instrumentos. A análise das correlações dos grupos do presente estudo demonstrou que houve uma correlação positiva baixa $(r=0,21)$, da variável $C O P$ com o fator $\mathrm{F} 3$ (conversação e desenvoltura social), evidenciando uma tendência de que, à medida que aumenta esta habilidade, aumenta a atitude cooperativa. Do mesmo modo, a variável $A G$ apresentou dados interessantes, pois demonstrou correlação negativa baixa $(r=-0,36)$ com GIHS (percentil Geral de Habilidade Social), o que indica que a habilidade social, em geral, diminui à proporção que a incidência de $A G$ tende a aumentar.

Nesse sentido, vale destacar que houve com a variável $A G$ do ZSC uma correlação negativa, significativamente moderada $(r=-0,49)$, com o fator F5 (autocontrole da agressividade em situações aversivas). Esta correlação é igualmente interessante, pois demonstra que, à medida que diminui o autocontrole da agressividade no IHS, aumenta a quantidade de respostas agressivas no Zulliger. Sendo a ideia principal desse estudo, verificar as evidências de validade do ZSC em correlação com os fatores do IHS, que teoricamente avaliam construtos similares, relacionamento e habilidade social, a correspondência dos resultados obtidos entre os instrumentos, fornece uma importante contribuição para a sua validade.

A variável GHR correlacionou-se positivamente baixa com três fatores do IHS, o GIHS (habilidade geral), F1 (Enfrentamento com risco) e F3 (conversação e desenvoltura social). Isso revela que, ao mesmo tempo em que aumentam essas habilidades sociais, também tende a aumentar o índice de respostas GHR. Contrariamente, a variável $P H R$ correlacionou-se negativamente baixa com três fatores, o GIHS, F3 e F5, indicando que, diferente de $G H R$, a variável $P H R$ diminui, à medida que a habilidade social geral, a de conversação e desenvoltura e a de autocontrole da agressividade aumentam. Ou seja, quando o indivíduo tende a fornecer respostas agressivas ou distorcidas, tende também a apresentar índices menores 
de habilidade social nestes fatores. Este achado corrobora com os resultados de Viglione et al. (2003), que afirmam que as variáveis $G H R$ e $P H R$ caminham em direção opostas.

A variável $P H R$, no entanto, correlacionou-se positivamente baixa com o fator $\mathrm{F} 1$ que está relacionado com o enfrentamento em situações de risco. Aparentemente contraditório nesta correlação, pode-se questionar que este índice F1 (enfrentamento com risco), apesar de ser uma habilidade social, está muito relacionado com a utilização da agressividade e de uma conduta mais assertiva.

A variável GPHR apresentou correlação positiva significativamente moderada com dois fatores do IHS, o fator geral GIHS e o de conversação e desenvoltura social F3. Isso sugere que a prevalência de respostas boas sobre as más caminha na mesma direção de aspectos mais maduros, ou elaborados, em habilidades de conversação e desenvoltura, e, habilidades sociais num âmbito geral.

Os conteúdos humanos, sendo importantes variáveis de relacionamento, obtiveram correlações significativas com o IHS. A variável SumH correlacionou-se positivamente baixa com os fatores GIHS, F3, F5 e obteve uma significância moderada com o fator F1, mostrando que, à medida que a pessoa possui um melhor repertório nas habilidades sociais, como de conversação e desenvoltura social, autocontrole da agressividade em situações aversivas e mais elaborado ainda na habilidade de enfrentamento com risco, aumenta a quantidade de respostas humanas, Sum $H$. As respostas $H$ puro que sugerem uma identificação clara e estável com pessoas (Villemor-Amaral \& Primi, 2009) obtiveram correlação positiva baixa com os fatores F5, e positiva moderada com F3, mostrando uma tendência de que, à medida que as pessoas deste grupo fornecem mais respostas humanas puras, aumentam o repertório da habilidade de autocontrole da agressividade e o repertório de conversação e desenvoltura social.

Importante lembrar, contudo, que para o desenvolvimento deste estudo foram selecionadas apenas as variáveis relacionamento do ZSC, consideradas fundamentais na proposta do trabalho. Assim, a presente discussão, além de promover auxílio na compreensão das questões sobre a dinâmica do indivíduo concernentes ao relacionamento interpessoal e a habilidade social, elucida e confirma uma das hipóteses iniciais em que se pretendia verificar se as variáveis que informam sobre o relacionamento e percepção interpessoal apropriada, $(C O P, G H R, G P H R, a$, Sum $H, H$ ) se correlacionariam positiva e significativamente com fatores do IHS. Portanto, se a obtenção de resultados compatíveis com os esperados, indica que o teste além de convergir com medidas similares, tem certo grau de especificidade (Primi et al., 2009), estes achados acumulam evidência de validade ao ZSC.

Em relação ao conteúdo $F d$, percebe-se que, apesar de poucas respostas, as fornecidas correlacionaram-se positivamente com os fatores GIHS, F1, F4 e F5. Para Zdunic (2003), as respostas de conteúdo comida aparecem pou- co, e ocorrem em protocolos com traços de dependência, mas podem apresentar-se em sujeitos "que vestem a camiseta", que se empenham em seus compromissos e se subordinam sem maiores comprometimentos.

É interessante destacar que a variável SumT demonstrou correlação positiva baixa com os fatores F1 e F2, indicando uma tendência de a pessoa dar maior sombreado textura, quando apresenta maior repertório na habilidade de enfrentamento com risco e na autoafirmação e expressão de sentimento positivo. Por outro lado, teve correlação negativa baixa e moderada com os fatores F4 e F5 respectivamente, demostrando que, quanto maior a habilidade de autoexposição a desconhecidos e situações novas, e também, maior o autocontrole da agressividade em situações aversivas, menor o número de respostas de sombreado textura $(\mathrm{Sum} T)$. Este dado vem de encontro aos estudos de Ferreira e Villemor-Amaral (2005) em que o relacionamento com pares e colegas em empresa correlacionou-se negativamente baixa $(r=-28) \operatorname{com} \operatorname{Sum} T$, onde se espera um trabalho profissional menos carregado de carências afetivas.

A variável $P E R$ apresentou aspectos interessantes observados na correlação negativa baixa com o fator F3, indicando uma tendência de que, à medida que a pessoa apresenta um bom repertório de conversação e desenvoltura social, diminuem as respostas personalizadas. Contrariamente, a variável $P E R$ indicou correlação positiva baixa com o fator F4 IHS, autoexposição a desconhecidos e situações novas, mostrando que, quanto melhor o repertório nesta habilidade, mais aumentam as respostas $P E R$. Este aspecto vem corroborar com o exposto por Zdunic (2003), onde se faz necessário o uso de uma atitude mais autoritária, pois, frequentemente, são encontrados valores $P E R$ elevados em vendedores exitosos que têm um estilo impetuoso de vendas e impõem seu próprio desejo de vender.

A variável Isolamento é considerada importante na análise da percepção do meio interpessoal e seu aumento pode estar relacionado com retraimento e isolamento social (Exner \& Sendín, 1999), além de a amostra ter apresentado um índice mais baixo, apontou uma correlação negativa baixa com os fatores gerais do IHS. Isso indica uma propensão de que, quanto maior o índice de isolamento, menor o repertório de habilidades sociais. Entretanto, observa-se, ainda, uma correlação positiva baixa do índice de isolamento com o fator F3 IHS, de modo que, à medida que apresenta um repertório mais desenvolvido de conversação e desenvoltura social, aumenta esta variável. Estes achados, inicialmente, parecem contraditórios, porém torna-se necessário salientar que o fator F3 correlacionou-se positivamente com COP, GHR, $G P H R$, Sum $H, H$, variáveis que indicam interesse interpessoal, e negativamente com PHR. Nesse sentido, a correlação positiva com o índice de isolamento parece estar mais relacionada à necessidade dos participantes de manter certo distanciamento nas relações interpessoais para evitar uma atitude mais invasiva. 
Em relação à variável $a-p$, que se refere aos movimentos ativos e passivos, a Tabela 3 apresenta indicadores negativos para correlações com os fatores GIHS, F3 e F5. Especificamente, os valores negativos estão relacionados com passividade, o que revela que, quanto mais aumenta a passividade, menor a habilidade social naqueles fatores. É necessário considerar que neste grupo, conforme a Tabela 1 houve uma tendência a uma atitude mais ativa, o que sugere uma capacidade de iniciativa e solução de problemas. Ferreira e VillemorAmaral (2005), encontraram correlações positivas entre movimento ativo e absorção, organização, registro e divulgação de conhecimento.

Torna-se evidente, portanto, que a hipótese inicial da presente pesquisa de que as variáveis que advertem sobre o relacionamento e percepção interpessoal prejudicada (AG, PHR, $p$, Sum T, PER, isolamento), correlacionar-se-iam negativa e significativamente com fatores do IHS, também se confirmou. Cumpre acrescentar que se o conjunto de evidências científicas contribui para assegurar as interpretações dos escores dos testes, e a relevância e utilidade dos usos propostos (Primi et al., 2009), os resultados ora discutidos, demonstram a importância da utilização do ZSC no âmbito empresarial.

\section{Conclusão}

Este estudo atendeu aos objetivos propostos, pois apresentou indicadores de evidências de validade para ZSC no contexto empresarial, como também a confirmação das hipóteses iniciais. A evidência da relação convergente entre o ZSC e os fatores do IHS que abordam construtos relacionados (relacionamento e habilidades sociais) igualmente, permitiu verificar as características de personalidade de indivíduos que exercem atividades empresariais na área de atendimento direto ao público.

Entre os indicadores, destacaram-se as correlações das variáveis $G H R$, GPHR, Sum $H$ do ZSC com os fatores GIHS, F3 e F1 do IHS, e as variáveis $A G, P H R$, $p$, Sum $T$ do ZSC com os fatores GIHS e F5. Os indicadores $G H R$, GPHR, Sum H, que informam sobre o bom relacionamento e percepção interpessoal adequada, correlacionaram-se positiva e significativamente com fatores GIHS, F3 e F1 do IHS. A variável $C O P$, apesar de não ter apresentado índices significativos de correlação com o IHS, apontou aspectos positivos qualitativamente, manteve-se na média normativa e em comparação à $A G$, mostrou-se superior. As variáveis $A G, P H R$, SumT, que podem informar sobre o relacionamento e a percepção interpessoal prejudicados, correlacionaram-se negativa e significativamente com os fatores GIHS e F5 do IHS. Esses achados foram considerados positivos, uma vez que apresentaram correlações expressivas em diversos indicadores do Zulliger, o que contribui para asseverar a sua validade no âmbito empresarial.

Os resultados deste estudo, ainda, devem ser compreendidos de forma preliminar considerando o fato de a amostra ter sido composta por um reduzido número de participantes. Porém, não se pode deixar de enfatizar que os principais achados atestam a validade do ZSC na investigação do relacionamento interpessoal, de profissionais que trabalham em empresa, o que confere ao instrumento propriedades necessárias para responder de forma segura nesse contexto.

\section{Referências}

American Educational Research Association, American Psychological Association, \& National Council for measurement in Education. (1999). Standards for educational and psychological testing. Washington, DC: American Educational Research Association.

Anastasi, A., \& Urbina, S. (2000). Testagem psicológica (7. ed.). Porto Alegre, RS: Artmed.

Bandeira, M., Costa, M. N., Del Prette, Z. A. P., Del Prette, A., \& Gerk-Carneiro, E. (2000). Qualidades psicométricas do Inventário de Habilidades Sociais (IHS): Estudo sobre a estabilidade temporal e a validade concomitante. Estudos de Psicologia (Natal), 5(2), 401-419. Retrieved September 15, 2009, from http://www.scielo.br/pdf/epsic/v5n2/ a06v05n2.pdf

Baumgartl, V. O., \& Primi, R. (2006). Evidências de validade da bateria de provas de raciocínio (BPR-5) para seleção de pessoal. Psicologia: Reflexão e Crítica, 19(2), 246-251.

Chiavenato, I. (2002). Recursos humanos. São Paulo, SP: Atlas.

Conselho Federal de Psicologia. (2003). Resolução 02/2003. Retrieved September 10, 2010, from http://www.pol.org.br

Del Prette, A., \& Del Prette, Z. A. P. (2005). Inventário de Habilidades Sociais (IHS Del Prete): Manual de aplicação, apuração e interpretação (3. ed.). São Paulo, SP: Casa do Psicólogo.

Del Prette, A., \& Del Prette, Z. A. P. (2006). Relações interpessoais no âmbito do trabalho e das organizações. Retrieved September 15, 2009, from http:// www.rihs.ufscar.br/treinamento-e-acessoria/copy_of_rihstrabalho

Dilchert, S., Ones, D. S., Visweswaran, C., \& Deller, J. (2006). Response distortion in personality measurement: Born to deceive, yet capable of providing valid assessments? Psychology Science, 48(3), 209-225. Retrieved October 6, 2006, from http://www.pabst-publishers.de/psychologyscience/3-2006/ps_3_2006_209-225.pdf

Exner, J. E., Jr., \& Sendin, C. (1999). Manual de Interpretação do Rorschach - Sistema compreensivo. São Paulo, SP: Casa do Psicólogo.

Ferreira, M. E. A., \& Villemor-Amaral, A. E. (2005). O teste de Zulliger e avaliação de desempenho. Paidéia (Ribeirão Preto), 15(32), 367-376. Retrieved November 30, 2008, from http://sites.ffclrp.usp.br/paideia/artigos/32/05.htm

Franco, R. R. C, Cardoso, L. M., Villemor-Amaral, A. E., \& Primi, R. (2009). Estudos de normatização, precisão e validade. In A. E. Villemor-Amaral \& R. Primi (Eds.), Teste de Zulliger no Sistema Compreensivo ZSC: Forma individual. São Paulo, SP: Casa do Psicólogo.

Franco, R. R. C., \& Villemor-Amaral, A. E. (2009). Ensaios de convergência entre provas de personalidade: Zulliger- SC e Pfister. (Tese de Doutorado não-publicada). Universidade de São Francisco, Itatiba, SP. 
Godoy, S., \& Noronha, A. P. P. (2005, jan./jun.). Instrumentos psicológicos utilizados em seleção profissional Revista do Departamento de Psicologia - UFF, 17(1), 139-159. Retrieved November 30, 2008, from http://www.scielo.br/ scielo.php? script=sci_arttext\&pid=S 0104 80232005000100011

Mahmood, Z. (1990, December). The Zulliger test: Its past and future. British Journal of Projective Psycology, 35(2), 2-16.

Meade, A. W. (2004). Psychometric problems and issues involved with creating and using ipsative measures for selection. Journal of Occupational and Organizational Psychology, 77, 531-552.

Muniz, M., Machado, M. A., Villemor-Amaral, A. E., \& Primi, R. (2009). Precisão do Zulliger no Sistema Compreensivo. In A. E. Villemor-Amaral \& R. Primi (Eds.), Teste de Zulliger no Sistema Compreensivo ZSC: Forma individual. São Paulo, SP: Casa do Psicólogo.

Pellini, M. C. A. (2008). Avaliação psicológica no campo da segurança publica e privada na atualidade. Trabalho apresentado no V Encontro da Associação Brasileira de Rorschach e Métodos Projetivos, Ribeirão Preto, SP.

Pianowski, G., \& Villemor-Amaral, A. E. (2008). O Teste de Zulliger na caracterização do transtorno obsessivo compulsivo. Estudos sobre aspectos técnicos e de aplicabilidade da avaliação psicológica [Resumo]. In Resumos do V Encontro da Associação Brasileira de Rorschach e Métodos Projetivos (p. 128). Ribeirão Preto, SP: Associação Brasileira de Rorschach e Métodos Projetivos.

Primi, R., Muniz, M., \& Nunes, C. H. S. S. (2009). Definiç̧ões contemporâneas de validade de testes psicológicos. In C. S. Hutz (Ed.), Avanços e polêmicas em avaliação psicológica (pp. 243-265). São Paulo, SP: Casa do psicólogo.

Santana, P. R., Cardoso, L., \& Villemor-Amaral. A. E. (2011). Instituindo a nota $\mathrm{Z}$ por meio do modelo de Rasch para o Teste Zulliger. Resumos do V Congresso Brasileiro de Avaliação psicológica: Avanços e Desafios. Bento Gonçalves-RS.

Silva, C. A. (2004). Habilidades sociais em fusão de organizações: Uma estratégia preventiva do estresse. (Dissertação de Mestrado não-publicada). Universidade Metodista de São Paulo, São Bernardo do Campo, SP.

Vaz, C. E. (2000). Atividades profissionais, "background cultural e interação social através da técnica de Zulliger forma coletiva". In E. C. Vaz, Técnica de Zulliger: Forma coletiva: Sistema Klopfer: Pesquisas básicas no Brasil. São Paulo, SP: Casa do Psicólogo.

Vaz, C. E. (2002). Z-teste: Técnica de Zulliger: Forma coletiva (2. ed. rev.) São Paulo, SP: Casa do Psicólogo

Viglione, D. J., Perry, W., Jarsak, D., Meyer, G., \& Exner, J. E. (2003). Modifyng the Rorschach human experience variable to create the human representational variable. Journal of Personality Assessment, 8(1), 64-73.

Villemor-Amaral, A. E. (2005). As pirâmides coloridas de Pfister. São Paulo, SP: Centro Editor de Testes e Pesquisas em Psicologia.

Villemor-Amaral, A. E. (2009). Métodos projetivos em avaliações compulsórias: Indicadores e perfis. In C. S. Hutz (Ed.), Avanços e polêmicas em avaliação psicológica (cap. 6). São Paulo, SP: Casa do Psicólogo.

Villemor-Amaral, A. E., \& Lamounier, R. (2006). Evidence of convergent validity of the EB in the Rorschach and Zulliger. Paper presented at the VII ERA Congress, Pádua, Italy.
Villemor-Amaral, A. E., \& Machado, M. (2008). O Depi e demais indicadores de depressão no Zulliger no Sistema Compreensivo. Estudos sobre depressão e técnicas projetivas [Resumo]. In Resumos do Simpósio do V Encontro da Associação Brasileira de Rorschach e Métodos Projetivos (p. 61). Ribeirão Preto, SP: Associação Brasileira de Rorschach e Métodos Projetivos.

Villemor-Amaral, A. E., \& Primi, R. (2009). Teste de Zulliger no sistema Compreensivo ZSC: forma individual. São Paulo, SP: Casa do Psicólogo.

Weiner, I. B. (2000). Princípios da interpretação do Rorschach. São Paulo, SP: Casa do Psicólogo.

Zdunic, L. A. (2003). El Teste Zulliger en la evaluación psicológica de personal. Aportes del sistema Compreensivo de Exner (2. ed.) Buenos Aires, Argentina: Paidós.

Zennaro, A., \& Lis, A. (1998, June). Fisher and Clevaland barrier and penetration scores for Italian adult and adolescent sample: Comparison of 2 versions scored on Zulliger test. Psychological Reports, 82(3, Pt. 2), 1411-1420. Retrieved November 21, 2009, from http://www.ncbi.nlm.nih.gov/ pubmed/9709543
Recebido: $11 / 01 / 2010$ $1{ }^{a}$ revisão: 04/10/2010 $2^{a}$ revisão: 09/12/2010 Aceite final: $16 / 12 / 2010$ 